Meta

Journal des traducteurs

Translators' Journal

\title{
La terminologie du Thésaurus : personne handicapée
}

\section{Sophie Janik}

Volume 34, numéro 4, décembre 1989

URI : https://id.erudit.org/iderudit/003252ar

DOI : https://doi.org/10.7202/003252ar

Aller au sommaire du numéro

Éditeur(s)

Les Presses de l'Université de Montréal

ISSN

0026-0452 (imprimé)

1492-1421 (numérique)

Découvrir la revue

Citer cet article

Janik, S. (1989). La terminologie du Thésaurus : personne handicapée. Meta, 34(4), 745-752. https://doi.org/10.7202/003252ar d'utilisation que vous pouvez consulter en ligne.

https://apropos.erudit.org/fr/usagers/politique-dutilisation/ 


\section{ÉTUDES TERMINOLOGIQUES ET LINGUISTIQUES}

\section{LA TERMINOLOGIE DU THÉSAURUS : PERSONNE HANDICAPÉE}

Cet article présente les lignes de force de la nouvelle terminologie contenue dans le Thésaurus: personne handicapée, paru à l'initiative de l'Office des personnes handicapées du Québec. Cette terminologie correspond à une nouvelle conception de leur situation dans notre société et se base sur la classification de l'Organisation mondiale de la santé concernant les déficiences, incapacités et handicaps. Cela nous donne également l'occasion d'amorcer une réflexion d'ordre théorique sur les descripteurs (langue documentaire) et les termes (terminologie).

\section{INTRODUCTION}

Depuis le début des années 80 , on assiste à l'échelle mondiale, à une évolution dynamique des concepts en matière de handicap. Les modèles traditionnels dans le domaine de la santé sont soumis à l'étude et critiqués car, on s'en rend compte, ils risquent de compromettre la compréhension du problème. On tend aussi à préciser de mieux en mieux les données statistiques concernant les personnes handicapées.

Selon l'Office des personnes handicapées du Québec (OPHQ) ${ }^{1}$, le taux de fréquence des personnes qui ont des déficiences physiques et mentales est de $7,4 \%$ de la population du Québéc. Les chiffres avancés par l'Organisation mondiale de la santé (OMS) font état de $10 \%$ de personnes handicapées dans les pays riches et $15 \%$ dans les pays pauvres ${ }^{2}$.

Dans les pays en voie de développement, il faut citer comme étiologies principales la poliomyélite, la lèpre, la tuberculose, les problèmes néo et périnatals ainsi que les parasitoses. Les conflits militaires et les conditions de vie précaire aggravent la situation générale de la santé des habitants de ces pays.

Dans les pays industrialisés, les causes dominantes sont les maladies cardiovasculaires ou respiratoires, ainsi que les accidents de la route, du travail, dans les sports... Il y a aussi le vieillissement des populations qui augmente le nombre des personnes dont les capacités d'adaptation sont de plus en plus diminuées : le Québec, dont le taux de natalité est l'un des plus bas à l'échelle mondiale, est particulièrement touché par cette réalité.

Dans le but de bien évaluer l'ampleur de cette problématique, l'OMS a élaboré et est en train d'expérimenter sa Classification internationale des déficiences, incapacités et handicaps. Pour sa part l'OPHQ a largement contribué depuis plusieurs années à améliorer la compréhension du processus d'apparition du handicap à partir des travaux de l'OMS ${ }^{3}$. La parution du Thésaurus: personne handicapée 4 (TPH) est le fruit de sa réflexion sur le plan terminologique.

\section{LE POURQUOI DU THÉSAURUS : PERSONNE HANDICAPÉE}

La publication, en 1966, du thésaurus en question, s'inscrit dans la formation principale de l'OPHQ qui est la coordination des services dispensés aux personnes handicapées. L'Office peut ainsi intervenir dans tous les secteurs pour assurer l'intégration sociale de sa clientèle. Il s'agit en l'occurrence d'organiser et de diffuser l'information relative à l'amélioration de la situation des personnes handicapées. 
En effet, le TPH précise le langage spécialisé concernant les personnes handicapées et l'enfance en difficulté. Ses 3697 descripteurs, avec leurs équivalents anglais répartis entre 95 schémas fléchés, permettent l'analyse et la recherche de la documentation écrite, audiovisuelle et du matériel tridimensionnel.

Rappelons brièvement que le thésaurus, ouvrage fondamental en documentation, contient une liste organisée et normalisée des descripteurs ou termes préférentiels. Ces mots ou groupes de mots sont censés présenter sans ambiguïté les notions contenues dans les documents et dans les requêtes de recherches documentaires. Le thésaurus est aussi un ouvrage terminologique puisqu'il propose un langage spécialisé relatif à un champ d'activité donné. Ajoutons également qu'un thésaurus à vocabulaire contrôlé possède, par sa définition même, une liste de non descripteurs ou termes rejetés qui portent le nom de synonymes documentaires.

Ainsi, concrètement, le TPH facilite l'organisation de nombreux centres de documentation spécialisés, permet la mise en place des banques de données, harmonise la terminologie utilisée et, par conséquent, coordonne les efforts de différents intervenants en facilitant les échanges d'information nationaux et internationaux.

Comme il s'agit d'un thésaurus à schémas fléchés ${ }^{5}$, procédé graphique hautement pédagogique puisqu'il permet d'un seul coup d'œil de saisir l'ampleur d'un champ sémantique, on peut s'en servir pour rédiger des textes, préparer des interventions ou tout simplement se familiariser rapidement avec la nouvelle terminologie.

De plus, l'index anglais des descripteurs constitue un précieux outil de travail pour tous ceux qui s'intéressent à la traduction.

Le THP propose, entre autres, une refonte du vocabulaire utilisé pour désigner les personnes handicapées qui accompagne nécessairement l'évolution de leur place dans notre société. La confusion règne encore dans les définitions ${ }^{6}$. C'est pourquoi il est impérieux de clarifier quelques notions fondamentales qui constituent la base de nos investigations.

\section{TERMINOLOGIE MÉDICALE VERSUS CLASSIFICATION INTERNATIONALE DES DÉFICIENCES, INCAPACITÉS ET HANDICAPS}

Les modèles traditionnels en matière de santé et plus précisément en ce qui concerne la compréhension du concept de la maladie (tel est par exemple le cas de la Classification internationale des maladies) mettent l'accent sur les pathologies en négligeant les personnes chez lesquelles elles apparaissent.

En effet, la terminologie médicale qui fait référence à l'étiologie ou à la localisation des lésions n'est pas suffisante pour rendre compte des difficultés persistantes rencontrées par les personnes qui ont des déficiences. Les signes et symptômes qui constituent le modèle médical de la maladie, bref, l'état clinique de la personne, doivent être mis en parallèle avec le contexte dans lequel la personne vit (famille, travail, éducation, loisirs, etc.) Il faudrait tenir compte d'une gamme complète de facteurs psychologiques et sociaux venant s'ajouter aux facteurs physiques. Dire qu'il y a au Québec quelques milliers de personnes qui sont hémiplégiques ne nous renseigne guère sur leurs besoins fonctionnels. Ces personnes rencontrent constamment des obstacles de toutes sortes qui entravent leurs possibilités d'apprentissage, leur recherche d'emploi, leur mobilité, etc. Certaines d'entre elles peuvent mener une existence tout à fait autonome alors que d'autres, à cause de divers facteurs, n'en sont pas capables.

Cette méconnaissance des besoins réels est due à l'absence de classification fonctionnelle dans l'épidémiologie médicale. C'est pourquoi une terminologie plus adéquate, utilisant un langage fonctionnel précis, a été proposée par l'OMS dans sa Classification internationale des déficiences, incapacités et handicaps ${ }^{7}$ (CIDIH). 


\section{COMMENT UNE PERSONNE DEVIENT HANDICAPÉE}

L'OPHQ, pour comprendre comment une personne devient handicapée, se sert des principaux concepts opératoires initialement définis par l'OMS dans l'original anglais de sa Classification et sa première traduction en français ${ }^{8}$. Quatre éléments distincts, mais reliés entre eux, y sont mis en valeur: la maladie (causes), la déficience, l'incapacité et le handicap.

\section{LES CAUSES}

Conformément à la politique d'ensemble À part... égale ${ }^{9}$ publiée par 1'OPHQ, il est préférable de parler des causes plutôt que des maladies puisqu'il s'agit d'un concept plus large et plus significatif.

Les causes pertinentes à la problématique du handicap sont celles qui provoquent une déficience. Elles peuvent être congénitales (la présence d'un chromosome supplémentaire provoque par exemple la déficience intellectuelle); pathologiques (la cataracte peut déclencher la déficience visuelle); traumatiques à la suite d'un accident, ou encore attribuables à un milieu à risque, etc.

Ainsi, on ne peut identifier les personnes handicapées à des personnes malades, mais la maladie peut avoir été à l'origine de leur situation.

\section{LA DÉFICIENCE}

Une déficience est une perte, une malformation ou une anomalie d'un organe, d'une structure ou d'une fonction mentale, psychologique, physiologique ou anatomique. Elle est le résultat d'un état pathologique objectif, observable, mesurable et pouvant faire objet d'un diagnostic. Une personne peut avoir une ou plusieurs déficiences à des degrés divers de gravité. La même déficience chez plusieurs personnes peut avoir différentes causes. Une déficience c'est par exemple la cécité, l'autisme, la paralysie cérébrale, etc.

\section{L'INCAPACITÉ}

La déficience se manifeste par une limitation d'une ou plusieurs fonctions. On est alors en présence d'une incapacité qui se définit comme suit : toute réduction (résultant d'une déficience), partielle ou totale de la capacité d'accomplir une activité d'une façon normale ou dans les limites considérées comme telles pour un être humain. Une incapacité ou une limitation fonctionnelle c'est par exemple une difficulté dans la communication orale, une incapacité de s'habiller seul, une maladresse dans les tâches manuelles.

\section{LE HANDICAP}

Un handicap est un désavantage social pour une personne, résultant d'une déficience ou d'une incapacité et qui limite ou interdit l'accomplissement de ses rôles sociaux (liés à l'âge, au sexe, aux facteurs socio-culturels). Le handicap d'une personne se définit donc à partir des obstacles qu'elle rencontre dans la société et qui empêchent son épanouissement et le développement de son autonomie. C'est une stigmatisation de la personne qui, en tant que porteuse d'une différence, est définie comme «anormale» par la société.

Ces distinctions permettent de mieux comprendre comment une personne devient handicapée et de clarifier les interventions à réaliser. Ainsi agir sur les causes, c'est faire de la prévention, agir sur les déficiences, c'est faire du diagnostic et du traitement, agir sur les incapacités, c'est faire de l'adaptation ou de la réadaptation et finalement agir sur les handicaps, c'est enlever les barrières sociales qui empêchent l'épanouissement et l'autonomie de la personne. 
Rappelons pour résumer que selon la Loi assurant l'exercice des droits des personnes handicapées ${ }^{10}$, une personne handicapée désigne «toute personne limitée dans l'accomplissement d'activités normales et qui, de façon significative et persistante, est atteinte d'une déficience physique ou mentale ou qui utilise régulièrement une orthèse, une prothèse ou tout autre moyen pour pallier son handicap».

\section{L'ÉVOLUTION DU VOCABULAIRE : DES INFIRMES AUX PERSONNES HANDICAPÉES}

Il n'y a pas si longtemps les termes comme infirme, fou, malade, aliéné, anormal faisaient partie de l'usage courant. Malheureusement pour les tenants d'une intégration à part égale des personnes handicapées, on retrouve encore de nos jours ce genre d'expressions négatives surtout dans la presse. Ce langage risque de nous ramener en arrière, d'entretenir les préjugés face aux personnes handicapées et de favoriser leur retour aux ghettos ${ }^{11}$. De plus, le langage usuel confond souvent des notions très différentes : il amalgame maladie et handicap, déficience intellectuelle et troubles mentaux.

Et pourtant au Québec, depuis une dizaine d'années, les associations de personnes handicapées et toutes sortes d'autres intervenants ont fait des efforts considérables pour introduire dans notre vocabulaire d'abord la notion de personne handicapée qui remplace celles de déficient, handicapé, infirme, invalide, et ensuite le concept de déficience, la distinction entre différentes catégories de déficiences: physique, intellectuelle, auditive, psychique, visuelle, etc.

Ces termes véhiculent l'idée, fondamentale quand une société cherche à intégrer un groupe minoritaire et marginalisé, qu'il s'agit de personnes, comme nous tous, avec toutes sortes de caractéristiques, tout comme nous tous, dont parfois une déficience... Comme cette dernière caractéristique varie beaucoup, il est plus juste de la préciser et de dire : personne ayant une déficience visuelle ${ }^{12}$ au lieu de aveugle, ou personne ayant une déficience motrice au lieu de infirme ou encore handicapé moteur.

Étiqueter et stigmatiser un être humain sans se soucier de sa personnalité, de ses capacités, de la situation qui lui est propre, constitue déjà une première source de handicap.

Dans le but de guider l'usager dans le choix des termes, un travail important sur le plan de non descripteurs a été accompli par les auteurs du TPH. Il s'agit ici de synonymes documentaires, c'est-à-dire des termes de forme différente, mais de sens voisin des autres, et pouvant être traités comme synonymes dans un langage documentaire donné. Ces termes qui ne doivent pas être employés pour représenter une notion, mais renvoient à un ou plusieurs descripteurs à utiliser à leur place, font partie du TPH et y sont même signalés aux deux endroits : une fois dans la liste des termes accompagnant chaque schéma fléché, une autre fois dans l'index permuté.

Sur 3967 descripteurs, il y a dans le Thésaurus: personne handicapée, 1963 non descripteurs. Cette synonymie est particulièrement abondante pour les termes en rapport direct avec les personnes handicapées.

Voici l'exemple de deux descripteurs, déficience intellectuelle et déficience auditive, qui battent tous les records quand au nombre de synonymes documentaires retenus:

\section{DÉFICIENCE INTELLECTUELLE}

EP (employer pour): arriération mentale arriéré mental débilité mentale déficient intellectuel handicap intellectuel

\section{DÉFICIENCE AUDITIVE}

EP : déficient auditif handicap auditif handicap de l'audition handicap de l'ouïe handicapé auditif hypoacousie 


\author{
handicapé intellectuel \\ oligophrénie \\ personne handicapée \\ intellectuellement \\ retardé mental
}

\author{
personne handicapée \\ auditive \\ perte d'audition \\ sourd \\ surdité \\ troubles auditifs \\ troubles de l'ouie
}

\section{LE CARACTÈRE NORMATIF DU THÉSAURUS : PERSONNE HANDICAPÉE}

L'examen rapide de l'organisation conceptuelle des descripteurs du TPH permet de constater que l'ensemble vocabulaire est divisé en plusieurs blocs. Nous partons de la problématique propre au développement de la personne, suivie de celles de la santé et du dépistage des déficiences. Viennent ensuite le traitement et les interventions d'adaptation ou de réadaptation, ainsi que le bloc concernant l'intégratión sociale des personnes handicapées.

L'utilisation de la classification de l'OMS pour l'élaboration d'une partie de ce vocabulaire est, selon nous, particulièrement intéressante. Son application aux fins de l'analyse documentaire constitue, dans l'état actuel de nos connaissances, une première mondiale ${ }^{13}$ et confère au TPH un caractère normatif.

Cette caractéristique peu orthodoxe dans le cas d'un thésaurus nous fournit l'occasion pour amorcer une réflexion théorique sur la nature d'un descripteur et d'un terme.

D'une manière générale, on retrouve dans le processus de communication trois formes linguistiques qui peuvent se distinguer l'une de l'autre. Ce sont les mots (langage usuel), les termes (terminologie) et les descripteurs ou termes d'un thésaurus (documentation) $)^{14}$.

De nombreuses convergences sont à signaler entre la terminologie et la documentation. Leur champ d'investigation est le même: il s'agit de structurer un ensemble de termes propres à un domaine spécialisé de la connaissance. L'objet particulier de leur recherche leur est également commun : les deux disciplines se penchent sur le concept et sa représentation matérielle et tentent de donner un sens univoque à chaque terme afin de faciliter l'échange d'information. Dans les deux cas, il s'agit de construire les langages artificiels, normalisés, contrôlés, et fortement réduits sur le plan syntaxique.

Le terme partage avec le descripteur la même définition classique du signe linguistique telle que proposée par $\mathrm{F}$. de Saussure. Après tout il s'agit de mots. Ce qui différencie le terme et le descripteur des signes linguistiques en général, c'est la démarche qui mène à l'identification du signifié (notion) et la priorité de cette dernière sur le signifiant (dénomination).

Cette situation est renversée dans la démarche lexicologique où, une fois la forme linguistique délimitée, on cherche à identifier un ou plusieurs sens qu'elle peut avoir. Voici la présentation schématique des deux démarches qui s'inspire des travaux de Guy Rondeau 15 :

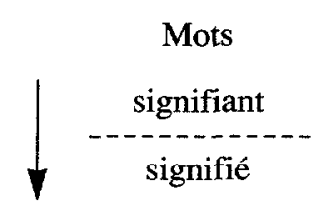

Terme descripteur

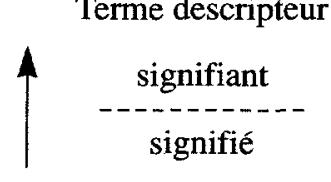


Autrement dit, pour identifier un descripteur ou choisir un terme, on part d'une notion qu'on connaît bien et pour laquelle il faut trouver une forme linguistique, ou encore choisir parmi plusieurs formes linguistiques données celle qui serait la meilleure. Notons également qu'un terme peut être créé, c'est-à-dire qu'une appellation nouvelle peut et doit être proposée dans le but de rendre compte de réalités nouvelles.

Tel n'est pas le cas pour le descripteur qui doit être choisi parmi les notions existantes et généralement acceptées. Il peut même arriver, dans le domaine de l'indexation, que pour un terme anglais on ne trouve pas d'équivalent français ${ }^{16}$. Pour résoudre ce genre de problèmes, des conditions plus favorables doivent se présenter.

Il pourra s'agir du développement de la recherche d'un consensus national ou international quant à la nomenclature d'un domaine ou encore de la création des néologismes propres à chaque pays qui est un travail strictement terminologique.

On décèle alors les différences ${ }^{17}$ entre la terminologie et les langages documentaires qui touchent d'abord la finalité : la terminologie assure la transition entre le discours scientifique et technique et l'univers de l'objet désigné, alors que dans le cas d'outil-thésaurus la fonction documentaire prédomine puisque, tout comme n'importe quel langage documentaire, il assure le lien entre l'information contenue dans les documents et les usagers.

Une autre différence concerne le contenu et la structure: présence de définitions dans les terminologies et leur absence dans les langages documentaires. On établit, entre les termes d'un thésaurus dont la signification est supposée connue des usagers, des relations sémantiques: associative, hiérarchique ou spécifique et celle d'équivalence. Le descripteur n'est donc «défini» que par la place qu'il occupe parmi d'autres descripteurs et le réseau de relations logiques qui s'y rattachent.

Enfin, la troisième divergence entre les deux disciplines est qu'un thésaurus n'est pas exhaustif, alors que la terminologie tend à l'être. Le thésaurus ne retient que les termes utiles au repérage des documents et il est de ce fait issu d'une terminologie beaucoup plus large et complète dans la description d'un domaine précis du savoir.

On voit que le TPH s'approche, au moins dans sa partie normative, des recherches relevant plus de la terminologie que de la documentation stricto sensu. Les notions fondamentales sont définies avec soin dans l'introduction du thésaurus où on explique en profondeur le parti pris terminologique et conceptuel.

Cette caractéristique pourrait être vue comme une faiblesse dans la confection même du TPH. En effet, n'étant pas d'une certaine façon passé par le feu de l'expérimentation au moment de sa publication, il devra subir l'épreuve de l'évaluation seulement après avoir été livré aux utilisateurs. Mais cela constitue également son originalité. Tout en s'appuyant sur une classification de plus en plus répandue dans le monde, le TPH véhicule les directives de l'OPHQ et sa volonté de modifier les habitudes langagières touchant la problématique des personnes handicapées. Déjà un réseau ${ }^{18}$ d'intervenants intéressés à la diffusion d'information relative à leurs clientèles cibles s'est constitué autour du thésaurus pour expérimenter et adapter, s'il y a lieu, la nouvelle terminologie à la réalité québécoise.

Compte tenu du rôle de l'OPHQ, le but principal de nos investigations est la meilleure compréhension du processus d'apparition du handicap qui sous-tend nos activités tant au niveau de la recherche que de l'intervention. Mais nos préoccupations d'ordre terminologique qui en découlent peuvent également être vues comme l'interventionnisme de l'État en matière de langue. Il ne s'agit pas ici de la mise en œuvre d'une politique linguistique ferme et entérinée par voie législative qui aboutirait à un réaménagement linguistique de tous les milieux de notre société - ce rôle incombe à l'Office de la langue 
française - mais plutôt de changement terminologique planifié propre à un domaine restreint qui constitue un cas particulier de changement linguistique en général.

L'OPHQ a d'ailleurs pris, lors de la conférence $\grave{A}$ part égale! en 1985, toute une série d'engagements ${ }^{19}$ concernant l'appellation, l'utilisation et la diffusion de la CIDIH. La publication du Thésaurus: personne handicapée en fait, par excellence, partie.

Il est certain que divers facteurs psychosociaux peuvent favoriser ou au contraire entraver l'implantation de la nouvelle façon de voir les personnes handicapées et d'en parler. Mais de nos jour, selon le mot de E. Brent ${ }^{20}$, «au lieu d'être inspiré par le souci strictement savant ou réformiste de quelques individus, le travail innovateur et normalisateur du terminologue s'inscrit (souvent) dans une politique gouvernementale».

Dans notre cas, elle vise à la fois à intégrer, sans discrimination ni privilège, les personnes handicapées dans la vie quotidienne de la communauté et à créer, via la diffusion de la nouvelle terminologie, des schémas linguistiques qui rendraient compte de la nouvelle organisation sociale. Ainsi, croyons-nous, les comportements linguistiques et, ce qui va de pair, les mentalités des locuteurs et locutrices pourront être transformés en profondeur.

SOPHIE JANIK

Office des personnes handicapées du Québec, Canada

\section{NOTES}

1. GADREAU, Rock (1984): Données statistiques sur les personnes ayant des déficiences physiques ou mentales au Québec en 1984, Drummondville, Office des personnes handicapées du Québec, p. 5.

2. Cité par Claude Hamonet (1984) : «La médecine de rééducation», Après-demain (numéro spécial intitulé «Les handicapés»), octobre, $\mathrm{n}^{\circ} 267$, pp. 13-17. Cependant ces chiffres dépendent de la façon dont on définit une personne handicapée.

3. Québec (Prov.), Secrétariat permanent des conférences socio-économiques (1981) : l'Intégration de la per. sonne handicapée. État de la situation, Québec, Secrétariat..., pp. 19-30 et avant tout Québec (Prov.), Office des personnes handicapées (1984) : $\dot{A}$ part... égale. L'intégration sociale des personnes handicapées: un défi pour tous, Québec, ministère des Communications, pp. 30-35.

4. JANIK, Sophie, Lise BRUNET et Louis-Luc LECOMPTE (1986): Thésaurus: personne handicapée. Thésaurus à schémas fléchés de descripteurs pour l'analyse et la recherche des documents et du matériel avec leurs équivalents en langue anglaise, Québec, Publications du Québec, 429 p.

5. On trouvera, dans le manuel d'usage qui précède les sections principales du Thésaurus : personne handicapée, l'illustration d'un schéma fléché, l'explication de son utilisation ainsi que des relations sémantiques établies entre les descripteurs qui y figurent.

6. JAMMAL, Amal, Geneviève LOSLIER et Annie GAILLANDRE (1986) : «L'épidémiologie et les mots pour le dire», META, 31:1, pp. 34-58. À la page 48 on peut lire que le mot invalidité évoque toute une série de termes comme: infirmité, incapacité, déficience, handicap. La différence entre ces termes, selon les auteures, est très ténue et elles sont tentées de l'appeler une différence «d'éclairage sémantique». Or, le modèle de l'OMS, dont notre article fait part, donne à certains de ces termes un sens nouveau et distinct. La partie synonymique (termes rejetés) du Thésaurus : personne handicapée peut aussi aider à comprendre la spécificité de ces notions.

7. Cette classification a d'abord été publiée en anglais: World Health Organisation (1980): International Classification of Impairments, Disabilities, and Handicaps. A Manual of Classification Relating to the Consequences of Disease, Geneva, WHO, 207 p. À ce jour deux versions françaises ont paru. Il s'agit de : Organisation mondiale de la santé (1981): Classification internationale des déficiences, des maladies, Paris, Institut national de la santé et de la recherche médicale, 229 p., et la version de 1984 portant le titre général de : Classification internationale des handicaps : déficiences, incapacités et désavantages, $170 \mathrm{p}$. 
8. La deuxième traduction française parue en 1984 a pour titre: Classification internationale des handicaps: déficiences, incapacités et désavantages. Le concept de désavantage y remplace celui de handicap au troisième niveau de la classification. Ce changement d'utilisation du concept de handicap qui fait aussi bien référence à la déficience ou à l'incapacité qu'au désavantage, entrâne beaucoup de confusion dans la compréhension du processus d'apparition du handicap. Pour ne pas compromettre la cohérence de son modèle d'intervention, l'OPHQ continue à soutenir «qu'une déficience et une incapacité n'entraînant pas nécessairement un handicap dans la mesure où l'organisation sociale est adaptée et les besoins spéciaux de soutien de la personne sont satisfaits». Voir Patrick Fougeyrollas (1985): Classification internationale des handicaps : déficiences, incapacités et désavantages ou Classification internationale des déficiences, incapacités et handicaps. Commentaires de l'Office des personnes handicapées du Québec, Drummondville, OPHQ, 3 p.

9. Voir note 3.

10. Loi assurant l'exercice des droits des personnes handicapées. L.R.Q., chapitre E-20, 1 à jour au 17 juillet 1986, Québec, Éditeur officiel, p. E-20.1/1(22).

11. Le Conseil de presse du Québec a été saisi, suite à la publication de deux articles dans un hebdomadaire de Lanaudière en août 1985 où, pour protester contre l'intégration de personnes handicapées dans le village de Rawdon, un journaliste employait des mots absolument négatifs pour parler des personnes handicapées ayant une déficience du psychisme : «fous lâchés lousses dans la ville... bande de zombies hypermédicamentés», etc. Le 26 février 1986 le Conseil de presse du Québec rendait une décision condamnant l'utilisation de propos «nettement préjudiciables» à l'égard des personnes handicapées.

12. Ce sont les termes les plus appropriés à l'heure actuelle pour désigner les personnes handicapées. Par contre il serait difficle de leur donner le statut des descripteurs car le langage documentaire, lui, n'a pas la même finalité que le langage parlé ou écrit. Pour résoudre ce problème, on propose dans le TPH l'utilisation d'une convention «t» introduite par la norme AFNOR 247-103, qui permet de combiner les descripteurs. Par exemple : «déficience auditive + parent» au lieu de parent sourd. Voir Association française de normalisation (1980): Thésaurus monolingues et multilingues. Symbolisation des relations: norme expérimentale $247-103,3 \mathrm{p}$.

13. Ce fait a été souligné en 1983 et 1985 lors de rencontres à Drummondville des intervenants étrangers chargés du dossier de l'OMS avec ceux de l'OPHQ.

14. FELBER, Helmut (1982): «Some Basic Issues of Terminology» in Incorporated Linguist, vol. 21, $\mathrm{n}^{\circ} 1$, pp. 12-24. On y trouve également une mise au point des différences les plus significatives entre la terminologie (terminological vocabulary) et la documentation (documentary thesaurus).

15. RONDEAU, Guy (1984) : Introduction à la terminologie, Chicoutimi, G. Morin, pp. 12 et 19-21.

16. Nous faisons ici l'abstraction de la problématique beaucoup plus large, celle de l'hégémonie de l'anglais au niveau international, bien que notre exemple en fasse partie. Il s'agit du terme «cross-lagged correlation» tiré du Thésaurus délinquance juvénile qui nomme une méthode statistique utilisée dans les pays anglophones, au Québec, mais pas en France. Au lieu de proposer un néologisme, tel d'ailleurs n'est pas le but d'un langage documentaire, les auteures dudit thésaurus, après la consultation des spécialistes, ont intégré tel quel ce terme dans le vocabulaire contrôlé français. Voir Vesna Balzina et Sophie Janik $(1980)$ : Thésaurus : délinquance juvénile, Montréal, Groupe de recherche sur l'inadaptation juvénile, Université de Montréal, $188 \mathrm{p}$.

17. MANIEZ, Jacques (1976): «Terminologies et thésaurus : divergences et convergences», in Association française de terminologie, Terminologie 76. Colloque international, Paris, La Défense, 15-18 juin 1976, Paris, la Maison du dictionnaire, c 1977.

18. JANIK, Sophie (1986): «Thésaurus : personne handicapée, outil de coordination des services documentaires spécialisés», présenté au Congrès conjoint ASTED-CLA (Association pour l'avancement des sciences et techniques en documentation et Canadian Library Association) le 23 juin 1986 à Québec. Cette communication a été publiée in Documentation et bibliothèques, vol. $33, \mathrm{n}^{\circ} 1,1987, \mathrm{pp} .11-17$.

19. FOUGEYROLLAS, Patrick (1985): Classification internationale des déficiences, incapacités et handicaps et modèle d'intervention sur le processus d'apparition du handicap au Québec. Les engagements de l'OPHQ concernant l'application, l'utilisation et la diffusion de la CIDIH lors de la conférence À part égale! les 27-28 février et 1er mars 1985, à Montréal, Drummondville, Office des personnes handicapées du Québec, 5 p.

20. BRENT, Edmond (1982): «Facteurs psychosociaux de l'implantation de nouvelles terminologies», in Fachsprache, vol. 4, no 3 , pp. 110-117. 